\title{
Identification des Strongles des Mulots et Campagnols décrits par Dujardin
}

\author{
par Marie-Claude DURETTE-DESSET \\ [Laboratoire de Zoologie (Vers) associé au C.N.R.S. (Pr A.-G. Chabaud), \\ Muséum d'Histoire Naturelle, 57, rue Cuvier, F. 75 - Paris, $\left.5^{\circ}\right]$
}

\begin{abstract}
Résumé
Au cours de recherches sur les Héligmosomes de France, nous avons été amenée progressivement à mettre en doute la nomenclature communément admise pour les espèces les plus fréquentes.

L'étude de dessins inédits de Dujardin, de récoltes faites à l'endroit où cet auteur avait travaillé, et de différents spécimens dus à l'obligeance de collègues étrangers, nous permet de rétablir la nomenclature qui était partiellement ou totalement inexacte pour les quatre espèces considérées.
\end{abstract}

\section{Summary}

Studies on unpublished drawings of Dujardin (1845), or on materials found in the place where this author had worked, and on specimens kindly lent by foreign colleagues, allow the author to modify the nomenclature of Heligmosomes, especially for 4 species collected in fied-mice and voles (Heligmosomum costellatum, Heligmosomoides polygyrum, Heligmosomoides laeve and Longistriata minuta).

A new subspecies, $H$. polygyrum corsicum, from Mus musculus brevirostris in Corsica, is described. 


\section{$I^{\circ}$ - Travail de Dujardin}

Dans son ouvrage fondamental de 1845, Dujardin éclaire la systématique des très petits Strongles qui vivent dans l'intestin des Rongeurs communs en France. Il découvre quatre espèces distinctes et les décrit de façon remarquablement précise. Il les sépare de la façon suivante :

— Le « Strongle à côtes obliques », Strongylus costellatus, a été trouvé deux fois chez le campagnol Arvicola arvalis. Il se reconnaît aux plis ou côtes obliques qui joignent la bande dorsale à la bande ventrale. Le mâle mesure $11 \mathrm{~mm}$, la femelle $16,8 \mathrm{~mm}$ (rapport longueur/largeur : $65 \mathrm{chez} \mathrm{le} \delta, 67 \mathrm{chez} \mathrm{la}{ }^{\circ}$ ). Les œufs sont longs de 100-110 $\mu$.

- Le «Strongle polygyre », Strongylus polygyrus, est très abondant et très fréquent chez le mulot Mus sylvaticus et a été trouvé plusieurs fois dans l'intestin du campagnol Arvicola arvalis. Le of mesure $7,1 \mathrm{~mm}$, la $\& 13 \mathrm{~mm}$; (rapport longueur/ largeur : 80 chez le $\hat{\delta}, 67 \mathrm{chez}$ la $\uparrow)$. Les œufs sont longs de 62-66 $\mu$.

— Le «Strongle lisse», Strongylus laevis, a été trouvé chez Arvicola subterraneus et une seule fois chez le lérot (Myoxus nitella). L'espèce existe aussi chez le mulot Mus sylvaticus, mais comme elle est assez difficile à distinguer de la précédente, Dujardin indique qu'il n'a pas toujours cherché à savoir laquelle des deux il avait sous les yeux, et ignore sa fréquence chez le mulot. Le ô mesure $4,5 \mathrm{~mm}$, la $\& 6,4 \mathrm{~mm}$ (rapport longueur/largeur : 43 chez le ${ }^{\star}, 53-56 \mathrm{chez}$ la ${ }^{\circ}$ ). Les $œ u f s$ sont longs de $74 \mu$.

— Le «Strongle nain », Strongylus minutus, est essentiellement parasite d'Arvicola sub:erraneus. Il a deux ailes membraneuses et une queue courte, conoïde. Le $\hat{\delta}$ mesure $2,25 \mathrm{~mm}$, la $\$ 2,4 \mathrm{~mm}$ (rapport longueur/largeur : $32 \mathrm{chez}$ le $\hat{\delta}, 33 \mathrm{chez}$ la $\uparrow$ ). Les œufs sont longs de $88-92 \mu$.

\section{$\mathrm{II}^{\circ}$ - Nomenclature actuelle}

A l'heure actuelle, nous récoltons chez les mulots et campagnols de France quatre espèces distinctes.

La détermination est rendue facile grâce à la monographie de Skrjabin, Schikhobalova et Schulz (1954), complétée sur certains points par les travaux récents de Tenora (1966). Nous obtenons les résultats suivants :

- Chez le mulot : Apodemus sylvaticus, une seule espèce est très commune : Heligmosomum skrjabini (Schulz 1926), qui, selon Tenora, a pour synonymes Nematospiroides dubius Baylis 1926, Sincosta aberrans Roé, 1929, Heligmosomum azerbaidjani Schachnasarova 1949 et Heligmosomum kratochvili Tenora et Barus 1955.

- Chez le campagnol : Microtus arvalis : Heligmosomum costellatum (Dujardin 1845) conforme à la description de Desportes (1943), mais non à celle des autres auteurs modernes. 
- Chez le campagnol : Microtus arvalis : Heligmosomum polygyrum (Dujardin 1845).

- Chez les campagnols : Pitymys subterraneus et Microtus arvalis : Longistriata wolgaensis (Schulz 1926).

\section{$\mathrm{III}^{\circ}$ - Difficultés rencontrées et solutions proposées}

Sur les quatre espèces de Dujardin, nous ne retrouvons donc ni minutus, ni laevis ; nous constatons que $H$. polygyrum qui, pour Dujardin, était l'espèce commune des mulots, désigne maintenant une espèce parasite des campagnols que nous ne trouvons pas chez les mulots; enfin, $H$. costellatum, récolté en France, est différent de l'espèce figurant sous ce nom dans les traités.

Après avoir vérifié ces résultats à Rennes (1), région où Dujardin a effectué ses études, nous en sommes venue à la conviction que des erreurs se sont progressivement introduites dans la nomenclature.

La nature des hôtes et surtout les caractères morphologiques publiés par Dujardin permettent de supposer que polygyrus, laevis et minutus de Dujardin correspondent respectivement dans la nomenclature actuelle à Heligmosomum skrjabini, $H$. polygyrum et Longistriata wolgaensis. Seul, $H$. costellatum a conservé une nomenclature exacte dans le travail de Desportes, mais non dans les monographies courantes.

Nous avons pu obtenir une certitude à ce sujet, car nous avons bénéficié, d'une part de l'envoi de spécimens récoltés et déterminés par nos collègues de Moscou (2), d'autre part de la communication des dessins originaux de Dujardin (3).

L'ensemble de ces documents confirme en tous points les hypothèses préliminaires et ne laisse aucun doute, car les figures de Dujardin sont d'une précision rigoureuse.

Il semble inutile de redécrire en détail chaque espèce, puisque, sous des noms variés, des descriptions modernes existent dans la littérature.

Nous nous bornerons donc à indiquer :

a) le ou les synonymes les plus importants; il est impossible de préciser cette synonymie lorsque, comme il est fréquent, l'auteur cite l'espèce sans indiquer la description sur laquelle il a fondé sa détermination;

b) par un bref historique, à quelle époque et pour quelle raison le nom a été détourné de sa signification originale ;

(1) Nous avons effectué une grande partie de ces récoltes dans les environs de Rennes, grâce à la précieuse collaboration de $\mathrm{M}$. J.-Cl. Beaucournu que nous tenons à remercier ici.

(2) Nous exprimons notre très vive reconnaissance à Mme N.P. Schikhobalova à qui nous devons plusieurs espèces particulièrement intéressantes.

(3) La collection d'helminthes de Dujardin semble perdue, mais il existe à la Faculté des Sciences de Rennes des documents inédits de cet auteur. Il s'agit de nombreux dessins non publiés se rapportant aux espèces décrites en 1845 , et sur lesquels figurent le nom d'espèce, le nom de l'hôte et la date de la récolte. C'est ainsi que Longistriata depressa, espèce type du genre, a pu être identifié par Desportes et Chabaud (1961). Nous exprimons toute notre gratitude à MM. les Professeurs Razet et Doby, grâce à qui nous avons eu communication des documents se rapportant aux Strongles de Rongeurs. 
c) les éléments les plus caractéristiques visibles sur les dessins originaux de Dujardin ;

d) les publications modernes où l'espèce est décrite de façon précise ; dans la mesure du possible, nous y ajoutons quelques figures pour préciser en particulier la disposition des arêtes cuticulaires et la structure des bourses caudales et des ovéjecteurs.

\section{$I V^{\circ}$ - Analyse des espèces}

A. Heligmosomum Costellatum (Dujardin 1845) Railliet et Henry 1909. Synonymies principales :

Les synonymies proposées pour cette espèce nous paraissent erronées. Ainsi que nous l'indiquons plus bas, $H$. halli (Schulz 1926) semble valide, et certaines espèces décrites comme $H$. costellatum se révèlent à l'analyse être des espèces distinctes.

\section{Historique :}

En 1909, en se fondant sur la description originale, Railliet et Henry choisissent Strongylus costeliatus comme espèce type du genre Heligmosomum.

Travassos e $i$ Darriba (1929) pensent redécrire l'espèce en se fondant sur un matériel d'Arvicola arvalis de Hambourg.

Cette description est reprise dans les principales monographies (Travassos 1937, Skrjabin et coll. 1954), mais ne correspond pas, comme nous le verrons plus bas, au c.stellatum de Dujardia.

C'est seulement en 1943 qu'un matériel qui paraît vraiment correspondre à la de:cription originale est retrouvé et décrit avec une grande précision par Desportes, mais l'au:eur, n'ayant pas de matériel de comparaison, croit que les différences qu'il relève entre ses observations et celles de Schulz (1926) d'une part, de Travassos et Darriba d'autre part, sont inhérentes à des différences d'interprétation.

\section{Dessins de Dujardin :}

Deux petites planches portent l'indication : «Strongylus costatus de l'estomac et de l'intestin du campagnol. 8 mai 1843 - 6 mai 1844 ». Le système des arêtes cuticulaires est figuré de façon extrêmement précise. L'extrémité postérieure de la $\%$ et l'ovéjecteur sont particulièrement détaillés. La bourse caudale du $\hat{\delta}$ n'est représentée que repliée sur elle-même, et les côtes ventrales sont seules bien distinctes.

Tous les éléments concordent avec la redescription donnée par Desportes.

\section{Données morphologiques modernes :}

Nous ne pouvons pas actuellement compléter l'étude morphologique publiée par Desportes, car le matériel que nous possédons n'a pas permis d'obtenir de bourse caudale bien étalée. Nous disposons cependant de dessins inédits de cet auteur où l'extrémité caudale du $\hat{\delta}$ est figurée en vue dorsale. La base des côtes externo-dorsales est assez caractéristique, car elle est enflée en boule à la naissance. En outre, chaque 

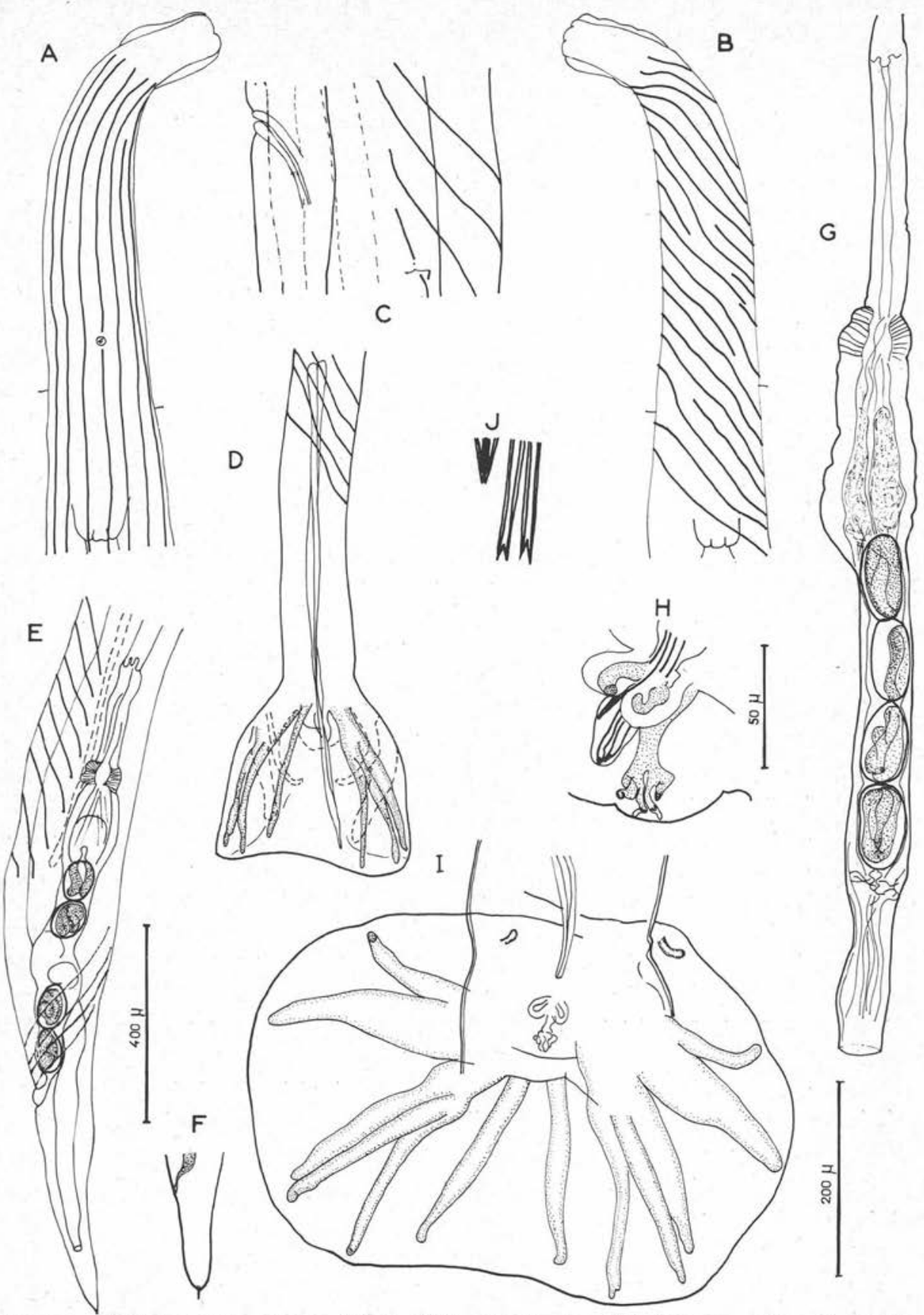

FIG. 1. - Heligmosomum mixtum Schulz 1927, parasite de Clethrionomys rutilus de Russie.

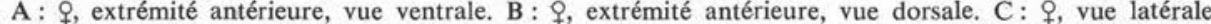
gauche, au niveau du pore excréteur. D : $\delta$, extrémité postérieure, vue dorsale. E: $ᄋ$, avec torsion postérieure du corps. Les 4 lignes obliques au-dessus de l'anus ne sont pas des arêtes, mais des plis cuticulaires résultant de la torsion du corps; la double ligne en tirets correspond à la ligne latérale droite. $\mathrm{F}: \mathcal{Q}$, queue, vue latérale gauche. $\mathrm{G}: \mathcal{Q}$, ovéjecteur disséqué. $\mathrm{H}: \delta$, détail du cône génital et de la côté dorsale. I : $\delta$, extrémité postérieure, vue ventrale. J : $\delta^{\star}$, détail de l'extrémité des spicules, schématisé. A, B, F, G, I : éch. : $200 \mu ; \mathrm{C}, \mathrm{H}$ : éch. : $50 \mu$; E : éch. : $400 \mu$ 
externo-dorsale possède une petite branche interne à son $1 / 4$ postérieur, qui s'anastomose avec sa symétrique sur la ligne médio-dorsale. La pointe caudale de la $q$ est conique, aiguë, mais n'a pas d'épine terminale.

Il semble y avoir plusieurs espèces très proches du véritable costellatum :

a) Heligmosomum halli (Schulz 1927). Contrairement à Desportes, nous pensons que cette espèce, parasite de Microtus arvalis aux environs de Moscou, est une espèce valide, car la pointe caudale de la i est tronquée et munie d'une pointe terminale ; en outre, la base des côtes externo-dorsales n'est pas enflée.

b) Heligmosomum mixtum Schulz 1954, in Skrjabin, Schikhobalova et Schulz 1954 ( $=H$. costellatum sensu Travassos et Darriba 1929).

Dans la principale monographie actuelle (Osnovi Nematodologie, vol. IV), Skrjabin, Schikhobalova et Schulz 1954 fondent la description d'H. costellatum sur les dessins publiés par Travassos et Darriba 1929, et non sur ceux de Desportes 1943.

Grâce à l'amabilité de $\mathbf{M}^{\mathrm{me}}$ Schikhobalova, nous disposons de spécimens provenant de Clethrionomys rutilus de Russie, étiquetés $H$. costellatum par nos collègues soviétiques. Ces spécimens (fig. I et II) correspondent en effet à la description de Travassos et Darriba, mais si nous les comparons aux spécimens français, nous nous apercevons immédiatement qu'il y a deux espèces différentes, car sur le matériel russe, comme sur celui de Hambourg, les stries cuticulaires sont obliques sur la face dorsale, mais peu visibles (car longitudinales) sur la face ventrale.

H. costellatum sensu Travassos et Darriba 1929 n'est donc pas $H$. costellatum sensu Dujardin 1845.

Pour désigner l'espèce de Travassos et Darriba, nous pensons pouvoir éviter 1a création d'un nom nouveau en utilisant le nom d'Heligmosomum mixtum. Cette espèce fut donnée comme nomen nudum par Schulz en 1951 dans l'Opredelitel parasititcheskich nematod, vol. III (avec, par erreur, la date de 1929). Dans l'Osnovi Nematodologie, vol. IV, l'espèce est décrite sous le nom de $H$. mixtum, Schulz, 1954, sur le seul exemplaire femelle dont dispose l'auteur.

Comme il s'agit précisément d'une espèce parasite de Clethrionomys rutilus de Russie, caractérisée par un système d'arêtes diagonales sur la face dorsale et longitudinales sur la face ventrale, nous pensons pouvoir faire l'assimilation à l'espèce décrite par erreur sous le nom de costellatum par Travassos et Darriba.

c) Nous décrirons prochainement des Héligmosomes de campagnols d'Alaska, extrêmement proches du costellatum, mais qui constituent cependant une espèce distincte.

d) Enfin, nous avons déjà, en collaboration avec Chabaud et Rausch, 1963, estimé qu'au Japon, chez Clethrionomys rufocanus et C. rutilus, l'Heligmosomum costellatum sensu Yamaguti 1954 n'est pas identique à celui de Dujardin, et nous l'avons nommé $H$. yamagutii. 

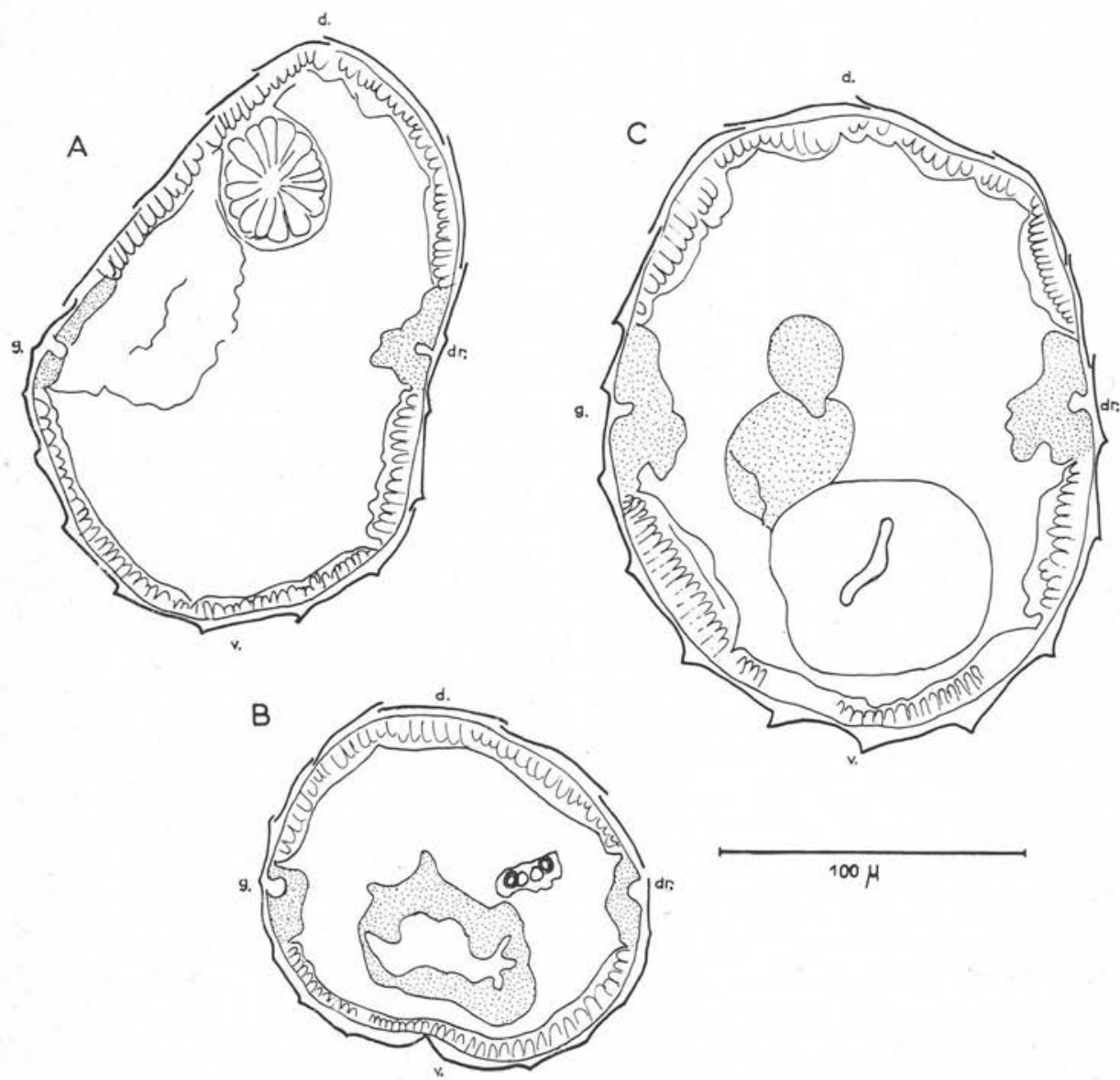

$100 \mu$

FIG. 2. - Heligmosomum mixtum Schulz 1927, parasite de Clethrionomys rutilus de Russie. Coupes transversales du corps. $\sigma^{2}: \mathrm{A}$ : à $4,9 \mathrm{~mm}$ en arrière de la tête. B : à $500 \mu$ en avant de la bourse caudale. $q$ : $\mathrm{C}$ : à $2,5 \mathrm{~mm}$ en arrière de la tête. La coupe passe par les glandes excrétrices

\section{B. HELIGMOSOMUM POLYGYRUM (DujaRdin 1845) R. et H. 1909.}

\section{Synonymies principales :}

Nematospiroides dubius Baylis 1926, Heligmosomoides skrjabini Schulz 1926;

Sincosta aberrans Roé 1929, Heligmosomum azerbaidjani Schachnazarova 1949;

Heligmosomum kratochvili Tenora et Barus 1955.

\section{Historique :}

Une première confusion résulte des travaux de von Linstow : en 1878, il décrit sous le nom de polygyrus une espèce du campagnol qui est certainement le laevis de 
Dujardin (il y a seize arêtes cuticulaires longitudinales; le schéma de la bourse caudale montre des côtes externo-dorsales non enflées à la base et une branche commune aux côtes latérales, courte. L'année suivante, il croit compléter la redescription précédente et le lecteur peut supposer qu'il s'agit du même matériel ou au moins du même hôte. En réalité, rien n'est précisé à ce sujet, et le schéma de la bourse caudale montre que Linstow a une autre espèce, qui, cette fois, correspond réellement au polygyrus (la base des côtes externo-dorsales est enflée, la branche commune aux latérales est plus longue).

En 1916, Hall interprète mal les figures de Linstow et crée, en se fondant sur les deux descriptions données par cet auteur, le taxon d'Heligmosomoides linstowi qui est donc synonyme pro parte de $H$. polygyrus et pro parte de $H$. laevis.

La description originale de Dujardin ne donnant pas de précisions suffisantes sur la bourse caudale, et l'espèce n'étant toujours pas redécrite, Hall (1916) et Travassos (1921) la classent à titre provisoire dans le genre Viannaia.

En 1922, Boulenger fait une excellente mise au point et donne une description très précise d'un Strongle récolté chez Microtus agrestis L. de la région de Birmingham. Par malchance, il l'identifie à polygyrus alors qu'il avait en sa possession le laevis de Dujardin.

En 1926, Baylis s'est rendu compte de l'erreur de Boulenger. Etudiant un Strongle récolté chez Apodemus sylvaticus de la région d'Oxford (qui est bien cette fois le véritable Strongylus polygyrus de Dujardin), Baylis est gêné par l'identification faite précédemment par Boulenger. Il énumère toute une série d'excellents arguments qui indiquent que c'est son espèce et non celle de Boulenger qui correspond au véritable polygyrus, mais, n'en ayant pas de preuve tout à fait décisive, il écrit : «In view of the insufficiency of Dujardin's description, and in order to avoid further confusion (pending a re-examination of the original material of $S$. polygyrus, if still in existence) it seems wisest to regard the form here described as a new species... » Il donne donc le nom de Nematospiroides dubius. Quinze jours plus tard, paraît le travail de Schulz où se trouve redécrite la même espèce sous le nom d'Heligmosomoides skrjabini. Après un échange de correspondance entre Baylis et Schulz, la synonymie est établie, et depuis vingt ans le polygyrus de Dujardin est désigné sous le nom d'Heligmosomum dubium (Baylis 1926) (4).

Nous n'insisterons pas sur quelques synonymies plus récentes, puisqu'une note de Tenora (1966) fait une mise au point de cette question et place en synonymie Sincosta aberrans Roé 1929, Heligmosomum azerbaidjani Schachnazarova 1949 et Heligmosomum kratochvili Tenora et Barus 1955.

(4) L'espèce parasite de Primates américains Longistriata dubia (Travassos 1921) a été décrite d'abord dans le genre Heligmosomum, et le taxon H. dubium a été pré-employé jusqu'en 1929, date à laquelle l'espèce de Primate fut placée dans le genre Longistriata. Le parasite du mulot pouvait donc légitimement, à partir de 1929 , être désigné comme $H$. dubium, la publication de Baylis ayant une légère priorité sur celle de Schulz. 


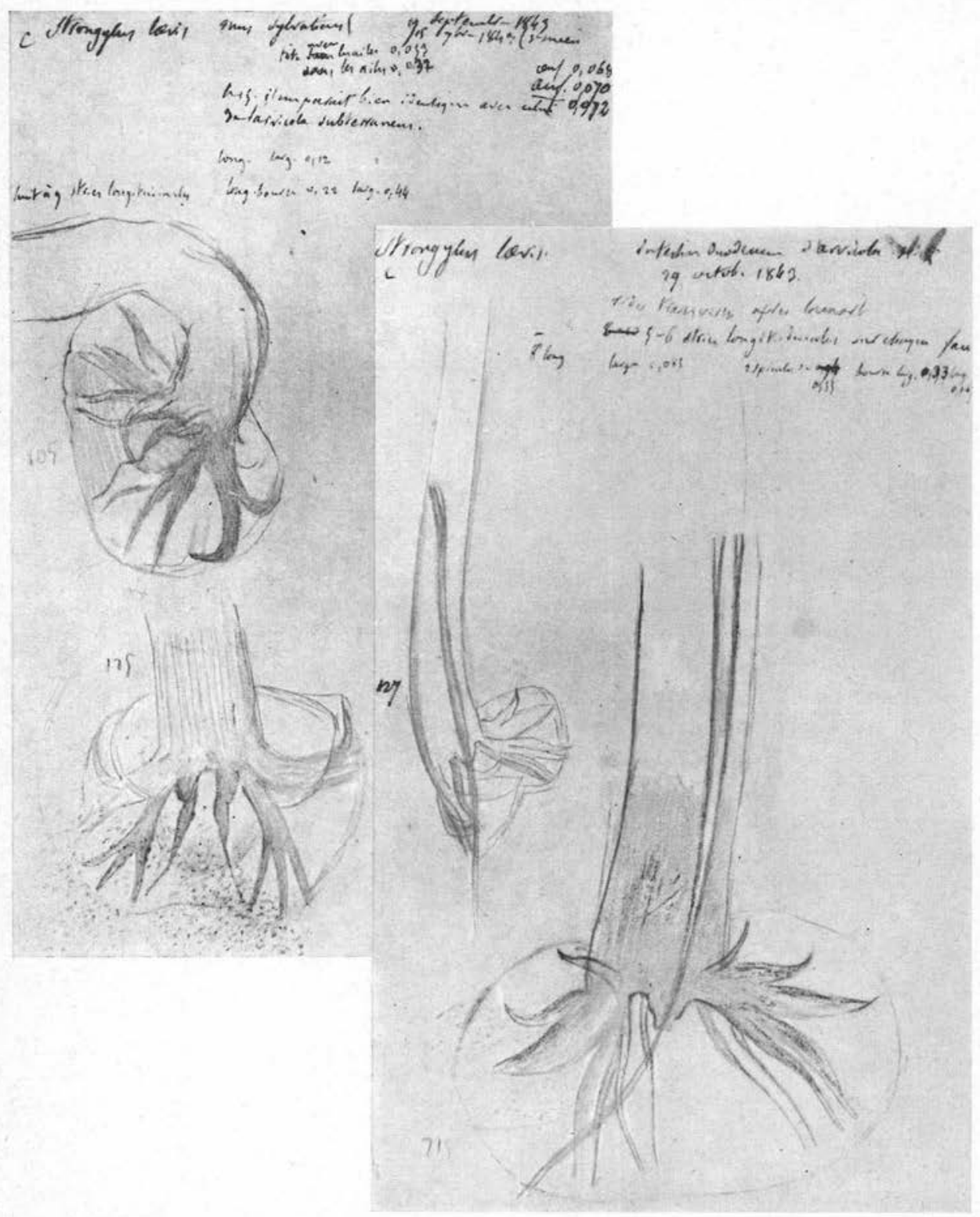

PLANCHE 1. - Reproduction de 2 planches de dessins originaux inédits de Dujardin. L'auteur n'a pas différencié nettement les mâles des 2 espèces. La planche gauche qui correspond à $H$. polygyrum, porte comme indication \& $C$. Strongylus laevis Mus sylvaticus ( 9 septembre 1843,19 novembre 1843. Tête avec les ailes: 0,053 ; sans les ailes : 0,037 ; œuf : 0,068 ; œuf : $0,070,0,072$. Bref, il me paraît bien identique avec celui de l'Arvicola subterraneus. Long.-larg. : 0,12 . Huit à neuf stries longitudinales. Long. bourse : 0,22 ; larg. : 0,44 . - - La planche droite qui correspond à $H$. laeve porte comme indication "C. Strongylus laevis, intestin duodenum d'Arvicola $\mathrm{n}^{\circ} 2.29$ octobre 1843 . Rides transverses après la mort. 5-6 stries longitudinales sur chaque face. $\delta^{2}:$ long. , large $0,085.2$ spicules de 0,55 . Bourse, larg. 0,33 , long. 0,167 » 


\section{Dessins de Dujardin :}

Dujardin a eu beaucoup de difficultés à séparer les deux espèces laevis et polygyrus, et les documents qu'il a laissés portent la trace de ses hésitations. Nous disposons en effet de neuf petites planches de dessins qui ont toutes été étiquetées primitivement «Strongylus laevis». Certaines d'entre elles sont surchargées de la main de Dujardin et portent l'indication «Polygyrus», d'autres portent simplement l'indication « B » qui correspond dans la rédaction définitive à ce qui est considéré comme polygyrus, l'indication « $\mathrm{C}$ » correspondant au contraire à ce que Dujardin décrit finalement sous le nom de laevis.

Pour séparer ses deux espèces, Dujardin a surtout utilisé les $q$ (longueur et mode d'enroulement du corps, taille des œufs, etc.) et il paraît s'être peu préoccupé des $\hat{o}$.

Il existe trois planches où figurent des bourses caudales, l'une du Srongle du mulot (pl. I à gauche), la deuxième de celui du campagnol des champs (pl. I, à droite), la troisième de celui du Pitymys. Toutes les trois sont déterminées Strongylus laevis \& $\mathrm{C}$ » et aucune n'est surchargée. Il semble que Dujardin n'ait pas réalisé nettement les différences entre les $\sigma^{*}$ de ses deux espèces, et ceci est d'autant plus curieux que deux des figures représentent des bourses caudales mises à plat et sont d'une qualité exceptionnelle.

$\mathrm{Si}$ ces dessins font savoir que Dujardin a bien eu les deux espèces entre les mains, ils ne permettent pas de préciser à laquelle des deux il a finalement attribué le nom de polygyrus. Cependant, grâce aux renseignements publiés, nous savons que polygyrus correspond pour lui à la grande espèce dont le corps est enroulé en une spirale serrée, espèce que nous trouvons actuellement chez les mulots de Rennes et non chez les campagnols. Nous devons donc déterminer comme polygyrus celle des planches de dessins qui se rapporte au Strongle du mulot. On y voit deux figures de bourses caudales mises à plat sur lesquelles se distinguent la dilatation de la base des externodorsales, le long pédoncule commun aux côtes latérales, l'asymétrie des lobes latéraux et tous les éléments qui caractérisent l'espèce désignée actuellement sous le nom d'Heligmosomum dubium (Baylis).

Nous pouvons donc affirmer, d'après ces documents, l'identité entre Strongylus polygyrus (Dujardin) et Heligmosomum dubium (Baylis).

\section{Données morphologiques modernes :}

Depuis les excellentes descriptions de Baylis et de Schulz, l'espèce a été étudiée de façon très détaillée par Roman (1951) (qui, comme Baylis, a fortement suspecté l'identité entre $H$. polygyrus et $H$. dubium).

Nous nous bornons à figurer les extrémités postérieures du $\hat{o}$ et de la $\uparrow$ et certaines coupes transversales du corps, d'après des spécimens provenant de la région de Rennes (France), parasites d'Apodemus sylvaticus (fig. III).

\section{Description d'Heligmosomum polygyrum corsicum n. subsp. :}

Heligmosomum polygyrum est une espèce extrêmement polymorphe, non seulement par la taille, mais encore par le nombre d'arêtes longitudinales et par l'aspect plus ou moins fortement asymétrique de la bourse caudale. Les papilles pré-bursales 


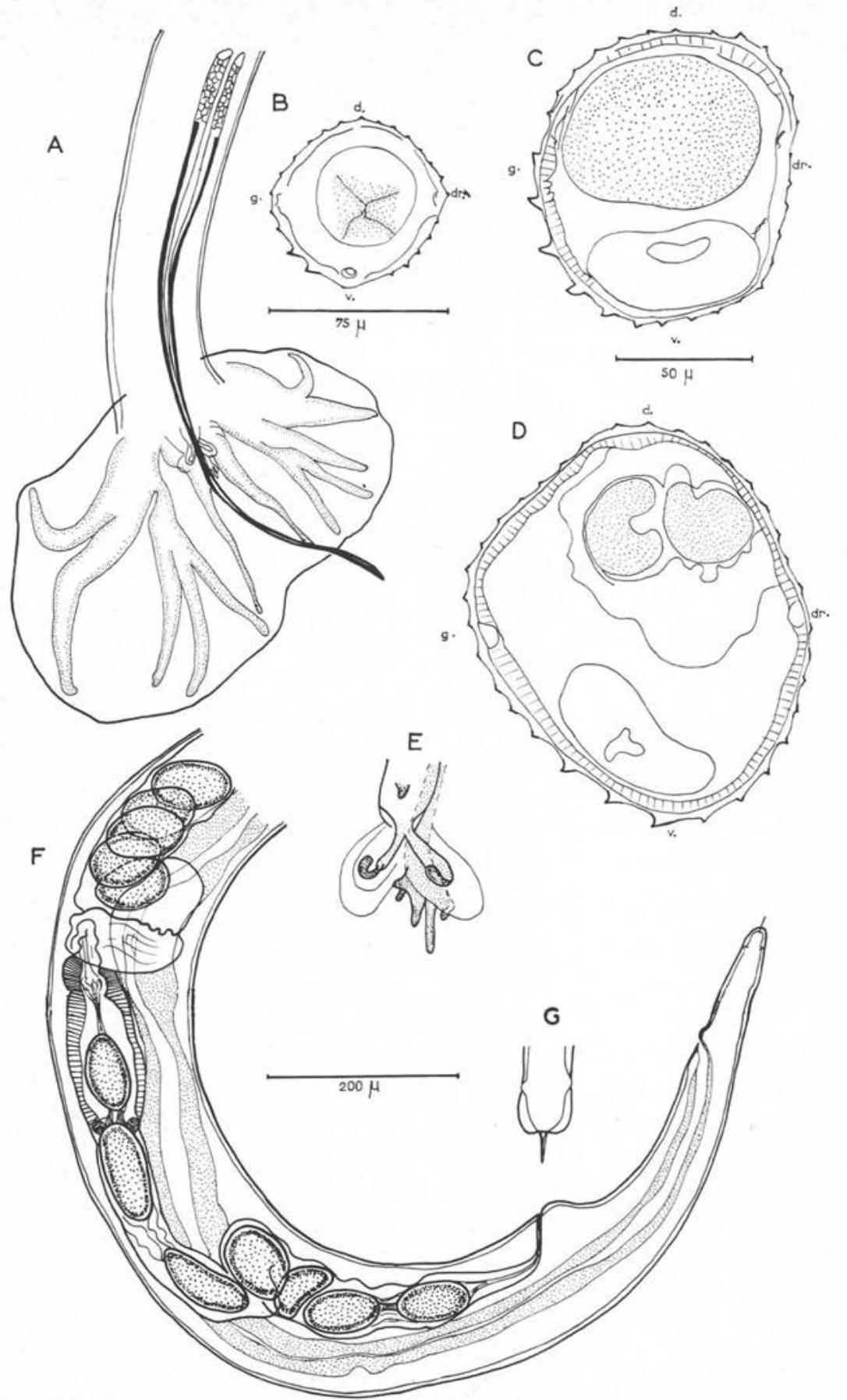

FIG. 3. - Heligmosomum polygyrum (Dujardin 1845), parasite d'Apodemus sylvaticus de la région de Rennes (France)

A : $\delta$, extrémité postérieure, vue ventrale. B, C, D: $ᄋ$, coupes transversales du corps au niveau du pore excréteur, au milieu du corps, à $3 \mathrm{~mm}$ en avant de la queue. E: détail du cône génital et de la côte dorsale. F : $q$, extrémité postérieure, vue latérale droite.. G :, , pointe caudale. A, F: éch. : $200 \mu ; \mathrm{B}, \mathrm{E}, \mathrm{G}:$ éch. : $75 \mu ; \mathrm{C}, \mathrm{D}$ : éch. : $50 \mu$

Annales de Parasitologie humaine et comparée (Paris), t. 43, 1968, $\mathrm{n}^{*}$ 
peuvent être très petites ou au contraire bien développées. Les côtes postéro-ventrales sont particulièrement variables, la droite dans sa longueur, la gauche dans son épaisseur. Nous n'avons pas réussi cependant, en comparant les spécimens de régions variées, à mettre en évidence de corrélations entre telle région et tel caractère, sauf dans le cas des parasites de la souris corse : Mus musculus brevirostris.
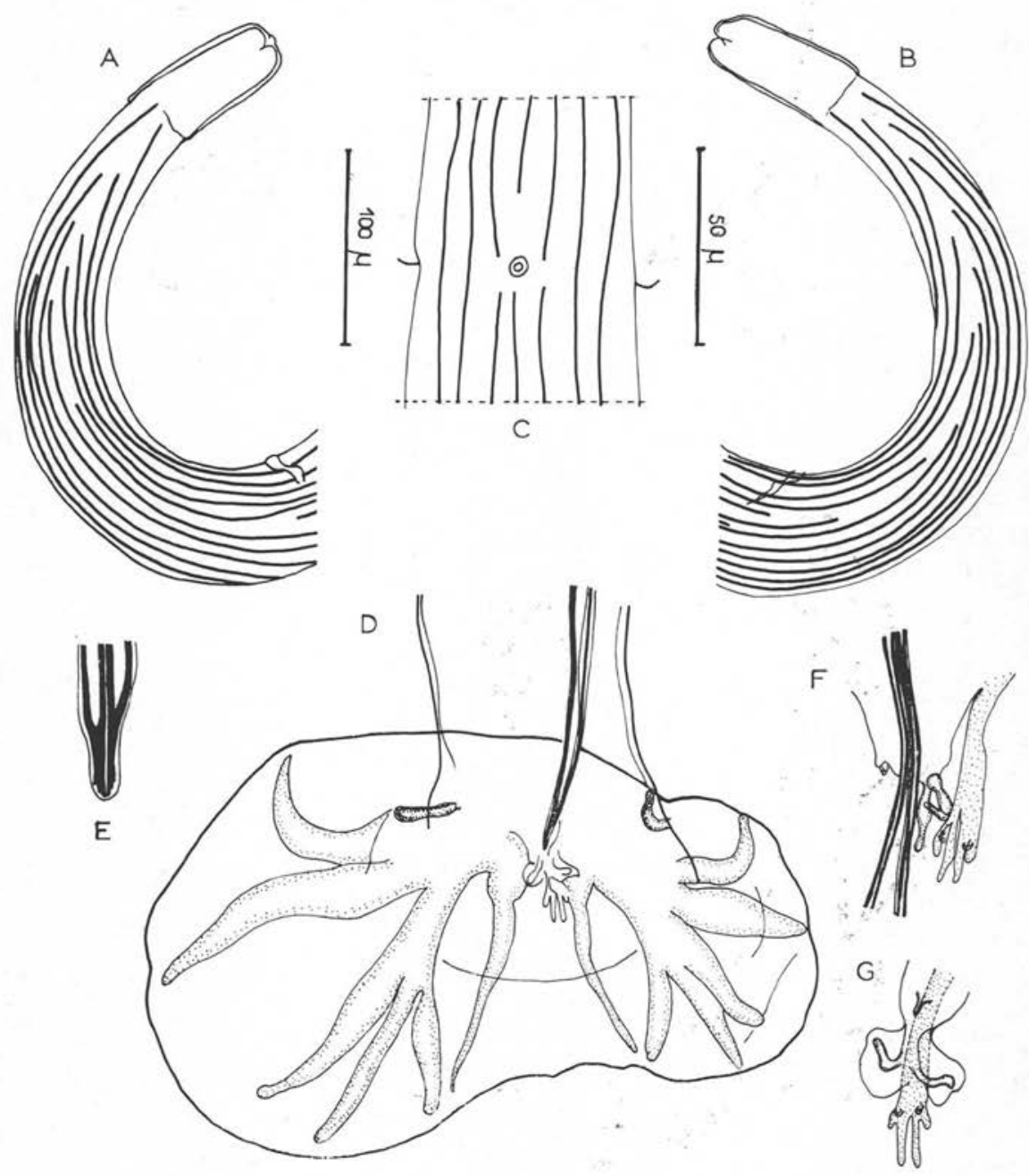

FIG. 4. - Heligmosomum polygyrum corsicum n. sub. sp., parasite de Mus musculus brevirostris de Corse. A : $q$, extrémité antérieure, vue latérale droite. B : $q$, extrémité antérieure, vue latérale gauche. $\mathrm{C}: \mathcal{Q}$, vue ventrale au niveau du pore excréteur et des deirides. D : $\delta$, extrémité postérieure, vue ventrale. E : extrémité des spicules. F : détail du cône génital et de la dorsale, vue latérale gauche. $\mathrm{G}$ : détail du cône génital et de la dorsale, vue ventrale. A, B, D : éch. : $100 \mu ; \mathrm{C}, \mathrm{E}, \mathrm{F}, \mathrm{G}:$ éch. $50 \mu$ 
Heligmosomum polygyrum est signalé à différentes reprises chez Mus musculus du continent, mais il y est relativement rare. Nous ne l'avons pas trouvé chez les souris sauvages des Baléares, et seulement une fois chez celles de France.

La fréquence chez les souris corses est au contraire importante, et la morphologie a, cette fois, des éléments particuliers.

Si l'on porte sur un graphique, en abscisse, la taille des spécimens, et en ordonnée le nombre des stries présentes dans la partie postérieure du corps, on constate que tous les spécimens provenant d'Apodemus, qu'ils soient originaires de Bretagne, d'Anjou, de Provence, des Iles Baléares ou de Corse, se situent au-dessous de la ligne en pointillés qui figure sur le graphique. Au contraire, les spécimens provenant de Mus musculus brevirostris sont tous au-dessus de cette ligne.

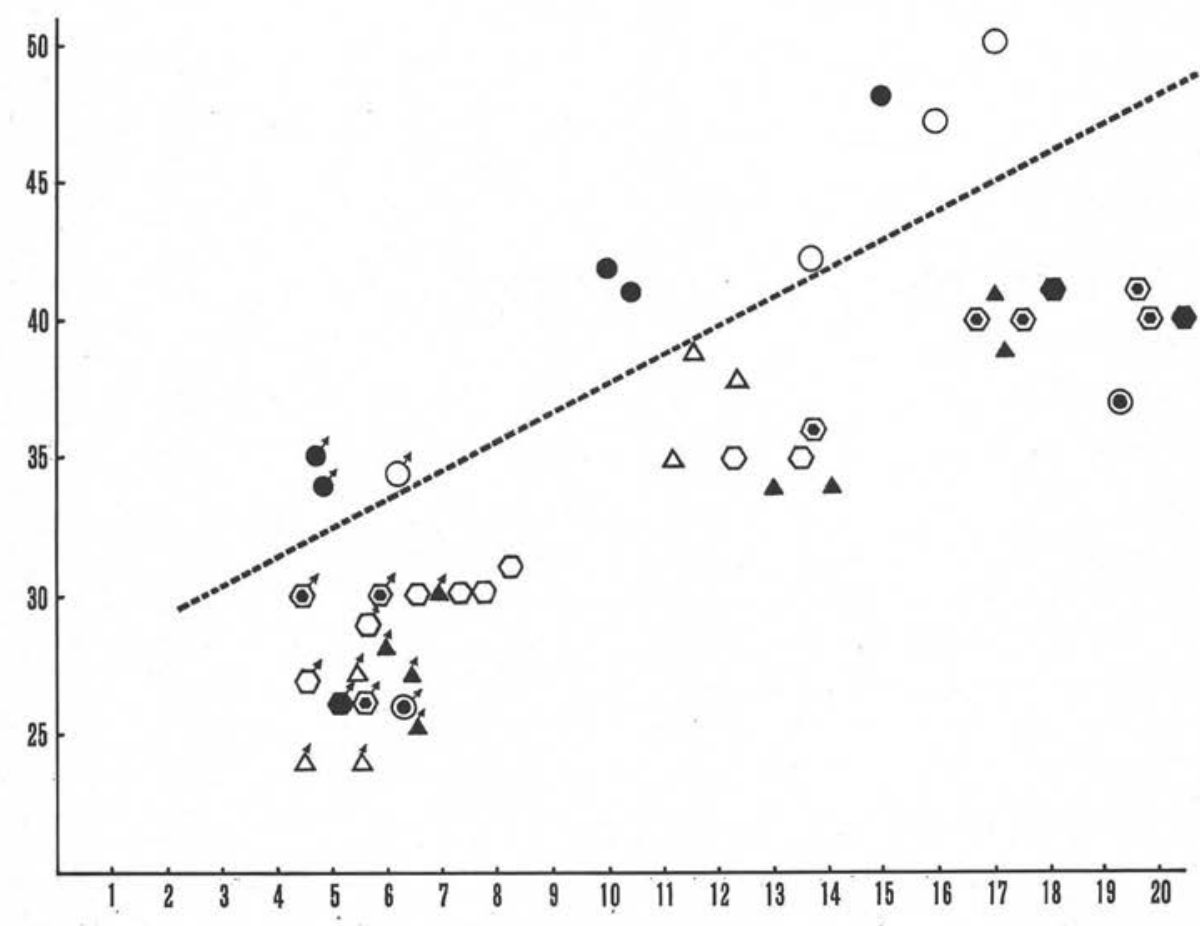

La longueur du corps est portée en abscisse, le nombre d'arêtes cuticulaires présentes dans la partie postérieure du corps, en ordonnée. Tous les spécimens d'Apodemus, qu'ils viennent du continent ou de Corse, et ceux de Mus qui viennent du continent, ont un nombre d'arêtes cuticulaires relativement faible par rapport à la longueur du corps. En revanche, tous les spécimens prélevés chez les Mus de Corse se trouvent situés au-dessus de la ligne en pointillés; le nombre des arêtes cuticulaires est relativement plus fort, seul élément pour définir la sous-espèce Heligmosomum polygyrum corsicum.

Signification des signes: Cercle blanc: Mus Corse (foie); cercle noir : Mus Corse ; cercle noir à l'intérieur d'un cercle blanc: Mus Région parisienne; triangle blanc: Apodemus Corse; triangle noir: Apodemus Baléares; hexagone blanc: Apodemus Nice; hexagone noir: Apodemus Richelieu; cercle noir dans hexagone: Apodemus Lavau-sur-Loire. Les signes surmontés d'une flèche corres-

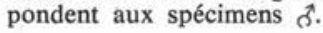


Nous estimons donc qu'il y a une faible spéciation chez les parasites de la souris corse et proposons le nom d'Heligmosomum polygyrum corsicum, nouvelle sous-espèce, définie seulement par une augmentation relative du nombre d'arêtes cuticulaires longitudinales (fig. IV).

On peut se demander pourquoi cette sub-spéciation ne se manifeste que chez les souris corses et non chez les mulots. On remarquera tout d'abord (fide F. Petter) qu'Apodemus sylvaticus sylvaticus du Continent et Apodemus sylvaticus dicrurus de Corse sont beaucoup plus proches l'un de l'autre que ne le sont Mus musculus du Continent et Mus musculus brevirostris de Corse. En outre, une observation évoque la possibilité d'une adaptation encore imparfaite du parasite chez cet hôte : c'est la localisation en plein parenchyme hépatique de plusieurs spécimens mûrs $\hat{\delta}$ et $q$ chez une souris capturée à Monacia, le 28 juillet 1965 .

C. HELIGMOSOMUM LAEVE (DUJARDin 1845) R. et H. 1909.

\section{Synonymies principales :}

Strongylus polygyrus (Dujardin), Linstow 1878 ;

Heligmosomoides linstowi Hall 1916 pro parte;

Heligmosomoides polygyrus (Duj.) Boulenger 1922.

\section{Historique :}

Ainsi qu'il a été dit ci-dessus, les espèces décrites par Linstow sous le nom de polygyrus sont: $H$. laeve, dans la publication de 1878 , et $H$. polygyrum, dans la publication de 1879.

Seurat (1916) a utilisé le nom pour un Heligmosome parasite de Dipodillus et de Meriones en Algérie, mais Travassos et Darriba (1929) ont relevé l'erreur et ont nommé l'espèce de Seurat Longistriata seurati.

Nous avons expliqué plus haut qu'en 1922, Boulenger a décrit sous le nom de polygyrus l'espèce correspondante au laevis de Dujardin. L'erreur s'est conservée depuis cette date et il faut donc, dans toutes les publications modernes, comprendre $H$. laeve lorsque l'auteur parle de polygyrus.

\section{Dessins de Dujardin :}

Une des planches, étiquetée Arvicola $\mathrm{n}^{\circ} 2$ (qui, d'après le contexte, correspond à Microtus arvalis), représente une vue ventrale de la bourse caudale étalée ; elle est d'une précision étonnante, superposable à la figure publiée par Boulenger en 1922 . Une autre planche, é.iquetée Arvicola subterraneus, est plus difficile à identifier, car il s'agit d'une vue ventrale d'une bourse caudale repliée. Il est possible cependant d'y reconnaître $H$. laevis et non $H$. polygyrum.

\section{Données morphologiques modernes :}

D'excellentes figures ont été publiées par Boulenger (1922) et Schulz (1926). Nous

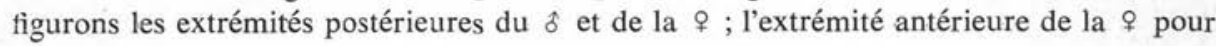



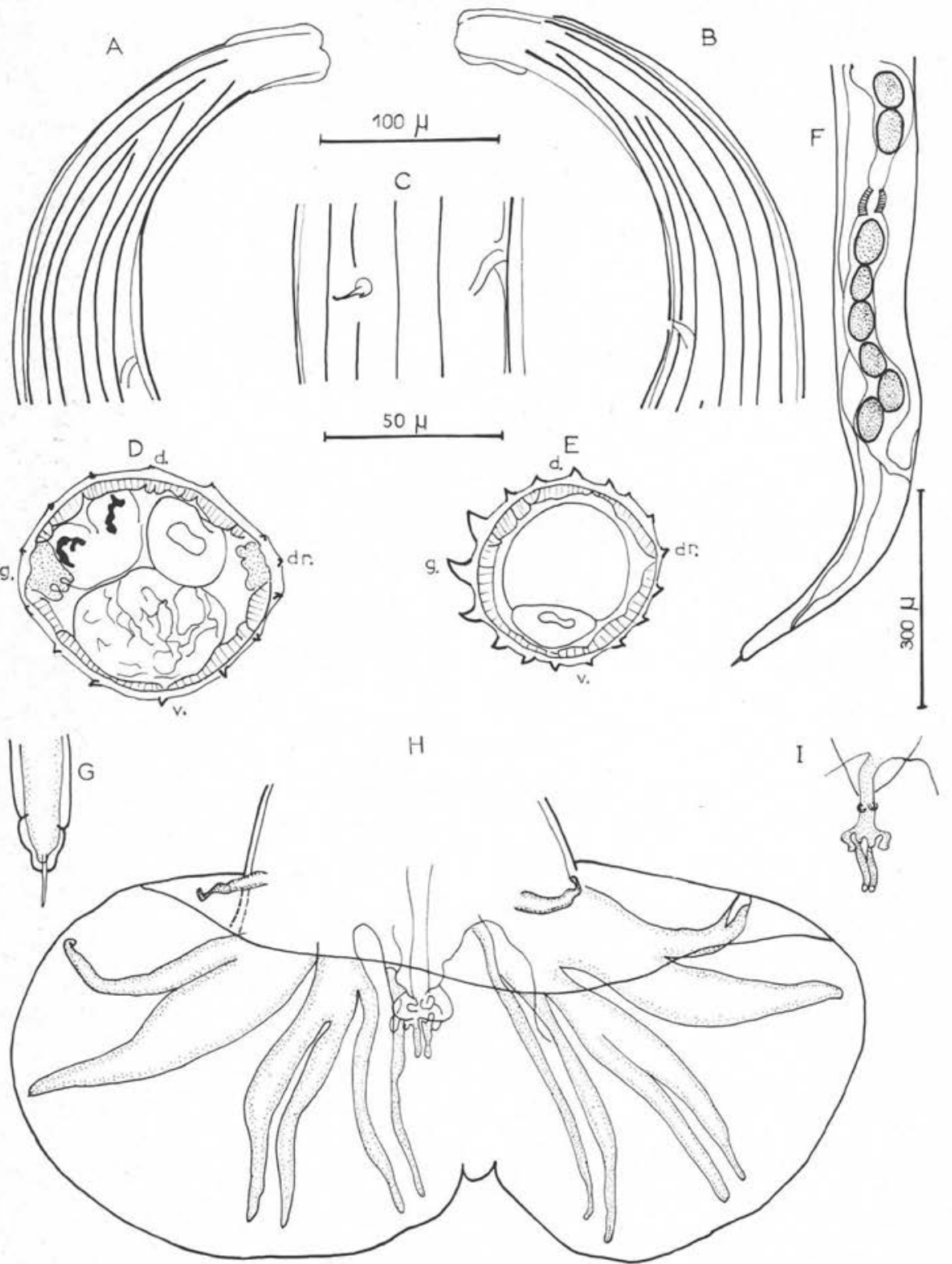

FIG. 5.- Heligmosomum laeve (Dujardin 1845), parasite de Microtus arvalis de la région de Rennes

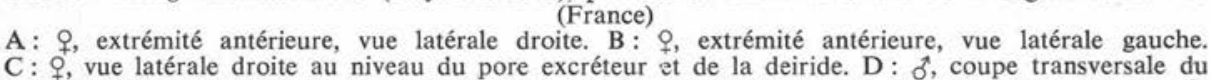
corps à $500 \mu$ en avant de la bourse caudale. E : $q$, coupe transversale au milieu du corps. F : $q$,

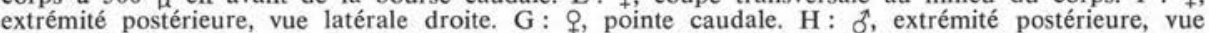
ventrale : I : $\delta$, détail de la dorsale. A, B : éch. : $100 \mu$; C, D, E, G, I : éch. : $50 \mu$; F : éch. : $300 \mu$ 
montrer le départ des arêtes cuticulaires, et des coupes transversales du corps d'après des spécimens provenant de la région de Rennes (France), parasites de Microtus arvalis (fig. V).

D. LONGISTRIATA MINUTA (DUJARDIN 1845) $n$. comb.

\section{Synonymies principales :}

Heligmosomum minutum (Duj.) Railliet et Henry 1909;

Longistriata wolgaensis Schulz 1926.

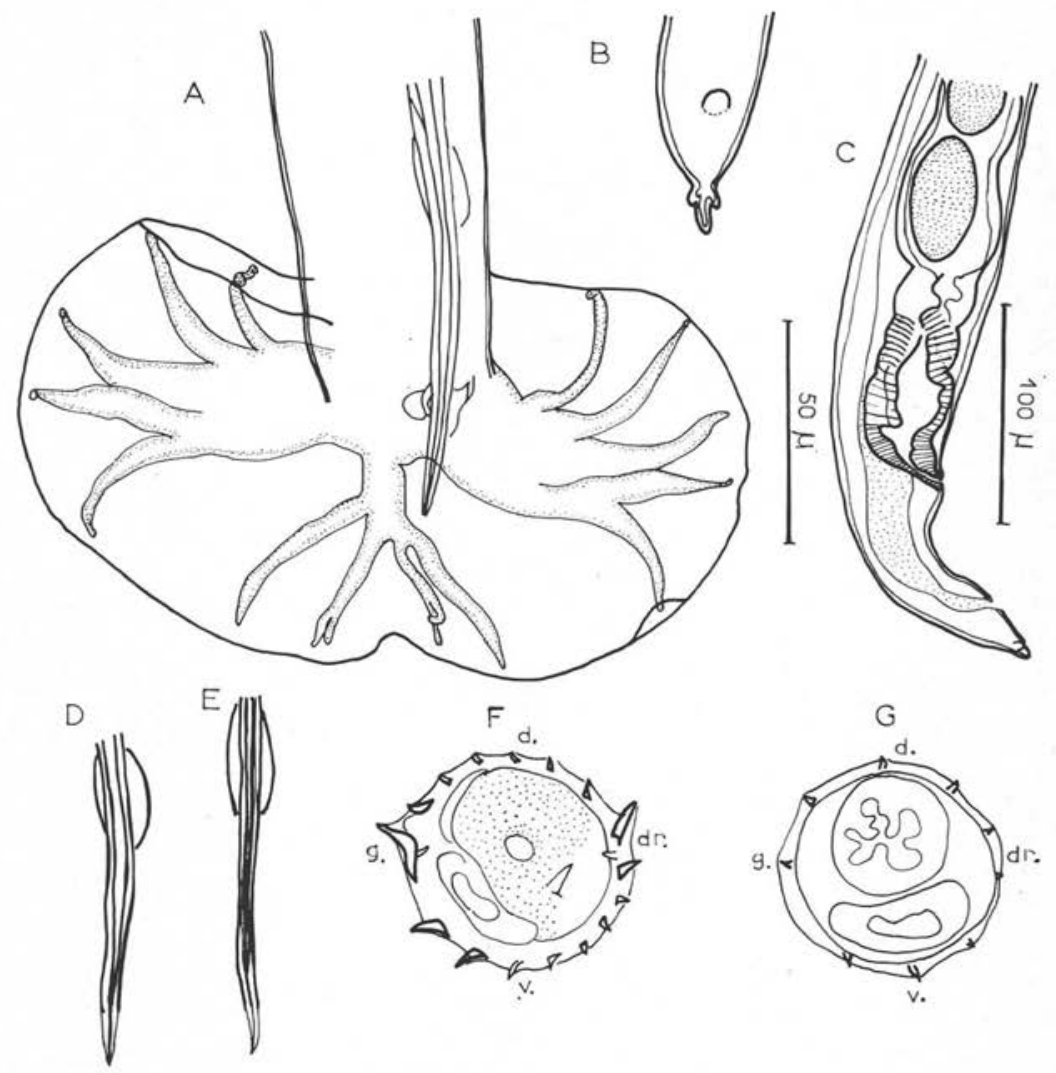

FIG. 6. - Longistriata minuta (Dujardin 1845) nov. comb., parasite de Pitymys subterraneus de la région de Rennes (France)

A : $\delta$, extrémité postérieure, vue ventrale. B: $\$$, extrémité caudale, vue ventrale. C: ${ }^{\circ}$, extrémité postérieure, vue latérale droite. D : extrémité des spicules, vue de face. E : extrémité des spicules, vue

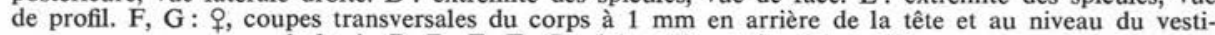
bule A, B, D, E, F, G : éch. : $50 \mu$; C: éch. : $100 \mu$

\section{Historique :}

Linstow (1882) a employé le nom de Strongylus minutus à mauvais escient pour un parasite de la taupe, erreur relevée par Hall (1916) et par Travassos (1921). 
Schulz (1926) semble être le premier à avoir redécouvert l'espèce chez Arvicola amphibius en Russie, mais il ne pouvait supposer qu'il s'agissait de l'espèce de Dujardin et il l'a considérée comme espèce nouvelle, sous le nom de Viannaia wolgaensis.

Ayant retrouvé l'espèce à Rennes chez Pitymys subterraneus et Microtus arvalis et ayant eu l'avantage de pouvoir examiner des spécimens donnés par nos collègues soviétiques, nous pouvons identifier $L$. wolgaensis à $L$. minuta.

\section{Dessins de Dujardin :}

Les dessins de Dujardin montrent en particulier l'extrémité postérieure de la bien caractéristique. La bourse caudale du $\hat{\delta}$ n'est figurée qu'à un faible grossissement et n'est pas très distincte, mais il est possible de voir toutes les côtes ventrales et latérales, et de longues côtes dorsales relevées au-dessus d'un lobe latéral.

\section{Données morphologiques modernes :}

La description de Schulz 1926 est très bonne et, depuis cette date, l'espèce a été facilement identifiée sous le nom de wolgaensis.

Nous figurons les extrémités postérieures du $\hat{o}$ et de la $\uparrow$, et des coupes transversales du corps, d'après des spécimens provenant de la région de Rennes (France), parasites d'un Pitymys subterraneus (fig. VI).

\section{Conclusions}

Grâce à des dessins inédits et à certaines recherches complémentaires, il est possible de rétablir la nomenclature des quatre Strongles de «mulots et campagnols » décrits par Dujardin en 1845.

1. Heligmosomum costellatum n'a été redécrit pour la première fois qu'en 1943 par Desportes. Les autres redescriptions publiées se rapportent à des espèces proches, mais différentes ; ainsi $H$. costellatum sensu Travassos et Darriba 1929 peut être assimilé à $H$. mixtum Schulz 1954.

2. Heligmosomum polygyrum était jusqu'à présent nommé $H$. dubium (Baylis 1926), H. skrjabini (Schulz 1926) ou H. azerbaidjani Schachnazarova 1949.

3. Heligmosomum laeve, à la suite d'une légère erreur de Boulenger (1922), (erreur soupçonnée par Baylis et Roman), était unanimement désigné sous le nom d'H. polygyrum.

4. Longistriata minuta $\mathrm{n}$. comb. était connu dans la littérature moderne sous le nom de Longistriata wolgaensis Schulz 1926.

Nous décrivons Heligmosomum polygyrum corsicum nov. subsp. chez Mus musculus brevirostris de Corse.

\section{Bibliographie}

BaYLis (H. A.), 1926. - On a Trichostrongylid Nematode from the wood-mouse (Apodemus sylvaticus). Ann. a. Mag. Nat. Hist., ser. 9, 18, 455-464.

Boulenger (C. L.), 1922. - The Structure and Systematic position of Strongylus polygyrus. Parasitology, 14, 206-213. 
Chabaud (A. G.), Rausch (R. L.) et Desset (M. Cl.), 1963. - Nématodes parasites de Rongeurs et Insectivores japonais. Bull. Soc. Zool. France, 88, 489-612.

Desportes (C.), 1943. - Un curieux Nématode, Heligmosomum costellatum (Dujardin, 1845). An. Parasitol., 19, 161-167.

- et Chabaud (A. G.), 1961. - Deux Trichostrongylides parasites de Musaraignes à Richelieu (Indre-et-Loire). An. Parasit., 36, 402-408.

Dujardin (F.), 1945. - Histoire naturelle des Helminthes ou vers intestinaux. Paris, XVI + $654+15$.

Hall (M. C.), 1916. - Nematodes parasites of Mammals of the Orders Rodentia, Lagomorpha and Hyracoidea. Proc. U.S. Nat. Mus. Wash., 50, 1-258.

Linstow (O.), 1878. - Neue Beobachtungen an Helminthen. Arch. Naturgesch., 44, 218-245.

-, 1879. - Helminthologische Studien. Arch. Naturgesch, 45, 165-188.

-, 1882. - Helminthologische Studien. Arch. Naturgesch., 48, 1-25.

Railliet (A.) et Henry (A.), 1909. - Sur la classification des Strongylidae et Metastrongylidae. C.R. Soc. Biol., 66, 85-88.

RoE (G.C.), 1929. - A new Nematode, Sincosta aberrans, new genus and species from a rodent. Proc. U.S. Nat. Mus. Wash., 75, 1-3.

Roman (E.), 1951. - Etude écologique et morphologique sur les Acanthocéphales et les Nématodes parasites des rats de la région lyonnaise. Mém. Mus. Nat. Hist. Nat., Paris, n.s., Sér. A, Zool., 2, 49-268.

Sachnazarova (S. S.), 1949. - Novyje nematody gryzunov Azerbejdzana. Trudy gelm. labor. An. SSSR, 2.

Schulz (R. S.), 1926. - Zur Kenntnis der Helminthenfauna der Nagetiere der Union SSR. I. Strongylata: 1. Fam. Trichostrongylidae Leiper, 1912. Trudy Gosudarstven. Inst. Experiment. Veter., 4, 3-30.

Seurat (L. G.), 1916. - Conditions de la ponte du Strongle lisse. Bull. Sc. France et Belg., $48,171$.

Skrjabin (K. I.), Schikhobalova (N. P.) et Schulz (R. S.), 1953. - Osnovi nematodologii. 4, Moscou, 1954, Acad. Nauk., SSSR, 323 p.

—, Schikhobalova (N. P.), Schulz (R. S.), Popova (T. I.), Boev (S. N.) et Deliamure (S. L.), 1952. - Opredelitel parasitichesk. Nematod., 3, Strongyliati, Moscou 1952, 890 p.

Tenora (F.), 1966. - Some remarks to the species of the genus Heligmosomum Railliet et Henry, 1909 with an asymetric bursa copulatrix. Folia Parasitologica, 13, 205-211.

— et Barus (V.), 1955. - Nalez cizopasne hlistice Heligmosonum skrjabini (Trichostrongylidae). Zool. a Entomol. Listy, 4, 51-54.

Travassos (L.), 1921. - Contribuiçao para o conhecimento da fauna helminthologica brasileira. XIII. Ensaio monographico da familia Trichostrongylidae Leiper, 1912. Mém. Inst. Osw. Cruz, 13, 5-135.

—, 1921 b. - Nematodeos novos. I. Brasil Med., an. 35, 2, 367-368.

—, 1937. - Revisao da familia Trichostrongylidae Leiper, 1912. Monogr. Inst. Osw. Cruz, I., $512 \mathrm{pp}$.

— et Darriba (A.), 1929. - Notas sobre Heligmosominae. Sc. Med., 7, 432-438.

Yamaguti (S.), 1954. - Studies on the Helminth Fauna of Japan. Part 51. Mammalian Nematodes, V. Acta Medicinae Okayama, 9, 105-121. 